\title{
Screening for Down's syndrome based on individual risk
}

\author{
Mark Lewis, Michael J W Faed, Peter W Howie
}

\author{
Abstract \\ Objective-To evaluate the effectiveness of bio- \\ chemical screening of individual pregnancies for \\ Down's syndrome risk.
}

Design-Retrospective determination of risk.

Setting-Obstetric and cytogenetic services in Tayside, Scotland.

Subjects - 3436 pregnant women who had screening for neural tube defects in the second trimester during November 1988 to March 1990 and whose pregnancies were dated by ultrasonography. Three women with pregnancies associated with Down's syndrome reported later in 1990.

Main outcome measures-Individual risk calculated from age at estimated date of delivery; chorionic gonadotrophin and $\alpha$ fetoprotein concentrations in serum samples obtained at precisely determined gestational ages in second trimester. Results of karyotype determination and outcome of pregnancy.

Results-During November 1988 to March 1990 karyotypes were determined for $5 \%$ of pregnancies for reasons of maternal age and genetic history and one of the eight affected fetuses was detected. Individual risk could not be calculated for 347 pregnancies, but screening on this basis would have detected five of the cases and required screening in 194 out of $3089(6 \cdot 3 \%)$ pregnancies; all three affected pregnancies reported later in $\mathbf{1 9 9 0}$ would also have been detected, giving a success rate of $73 \%(95 \%$ confidence interval $39 \%$ to $94 \%$ ). The age distribution of women according to individual risk suggests that women over 35 would be screened effectively.

Conclusion-Screening based on individual risk would use resources more effectively than screening based on maternal age and genetic history without affecting detection rates in older women.

\section{Introduction}

Trisomy 21 (Down's syndrome) is the commonest human chromosomal abnormality, occurring in about one in 700 babies.' Down's syndrome can be detected during pregnancy by determining the karyotype of fetal cells, but the limited resources available for this test and the morbidity associated with sampling fetal tissue restrict the procedure to women who, on the grounds of age or genetic history, are thought to be at highest risk.

The risk of conceiving a child with Down's syndrome increases sharply with age, and at present women aged 36 years at the estimated date of delivery are widely regarded as having a right to be offered karyotype determination. ${ }^{2}$ Only $30 \%$ of pregnancies associated with Down's syndrome occur in women over 35 years old, however, and in practice about $15 \%$ of cases are detected antenatally. ${ }^{3}$ In women with pregnancies associated with Down's syndrome mean concentrations of $\alpha$ fetoprotein, chorionic gonadotrophin, and unconjugated oestriol are abnormal for gestational age. ${ }^{4-6}$ Wald $e t$ al proposed that by modifying a woman's age related risk according to the concentrations of these biochemical markers the same number of karyotype analyses would be used more rationally, increasing threefold the proportion of affected pregnancies detected in the second trimester. ${ }^{3}$ They speculated that the greatest improvement in detection rate would be achieved by using $\alpha$ fetoprotein and chorionic gonadotrophin assays. We conducted a study to investigate this proposition.

\section{Subjects and methods}

We studied 3436 women who had been screened for neural tube defects in the second trimester of pregnancy during November 1988 to March 1990. This represented $58 \%$ of the total screened obstetric population in Tayside. All the women had had their pregnancies dated by ultrasonography. The serum samples obtained at screening were analysed for $\alpha$ fetoprotein and chorionic gonadotrophin blind to pregnancy outcome after the termination of the pregnancy or birth of the baby.

Concentrations of $\alpha$ fetoprotein were measured by an immunoradiometric assay, ' which was modified to allow magnetic separation. ${ }^{7}$ The between assay precision (coefficients of variation) during the study ranged from $10 \%$ at $8 \mathrm{kU} / 1$ to $5 \%$ between 30 and $500 \mathrm{kU} / 1$. Chorionic gonadotrophin was measured by a radioimmunoassay ${ }^{8}$ modified for routine use on serum specimens taken in the second trimester. The between assay precision ranged from $10 \%$ at $3 \mathrm{kU} / 1$ to less than $6 \%$ between 9 and $60 \mathrm{kU} / 1$. Reagents for these assays were produced on a large scale for the Scottish Health Service and are available from our laboratory and from the Scottish Antibody Production Unit at Law Hospital, Lanarkshire.

Maternal age was read from the women's unit numbers. The cytogenetic database, which contains a record of all karyotypes determined since January 1987, was searched for reports of trisomy 21 in amniotic fluids, products of conception, and neonatal specimens. Details on amniotic fluid specimens analysed between November 1988 and March 1990 were retrieved to determine maternal age, the reason for the karyotype determination, and the result of the analysis.

Gestational age at the time the mother's blood was sampled was calculated by the Alpha risk calculation programme (Logical Medical Systems), which uses biparietal diameter, date of ultrasonography, and regression parameters from the appropriate intrauterine growth chart. ${ }^{9}$

All these variables were used to estimate risk of Down's syndrome for a particular pregnancy by modifying the woman's age related risk with reference to the $\alpha$ fetoprotein and chorionic gonadotrophin concentrations in multiples of the appropriate median at the gestational age on the sample date. Alpha data files were also used to analyse the age and distributions of the study population and of the women who would have been offered screening on basis of this risk analysis. We also calculated risk of Down's syndrome in three women with affected pregnancies who had been screened for neural tube defects between April and December 1990.

\section{Results}

We estimated the risk of Down's syndrome for 3089 pregnancies; no risk could be estimated for 347 
pregnancies as the blood sample had been obtained before 105 days' gestation. After the risks for the first 1200 pregnancies had been calculated, the threshold for screening was set at a risk of one in 350 as at that stage $5 \%$ of estimates were higher than that value and karyotypes are determined for $5 \%$ of pregnancies with the current policy. With a threshold of one in 350 screening would have been offered for 194 of the total of 3089 pregnancies.

Figure 1 shows the distribution of calculated risks in relation to maternal age for the 3089 pregnancies. Nine pregnancies with a fetus with trisomy 21 were identified from the cytogenetic database. In one the karyotype was determined from a chorionic villus sample and so we could not estimate the risk. The eight remaining cases were included in the study and occurred in women less than 35 years old. In five cases the risk estimate was above the screening threshold (fig 1).

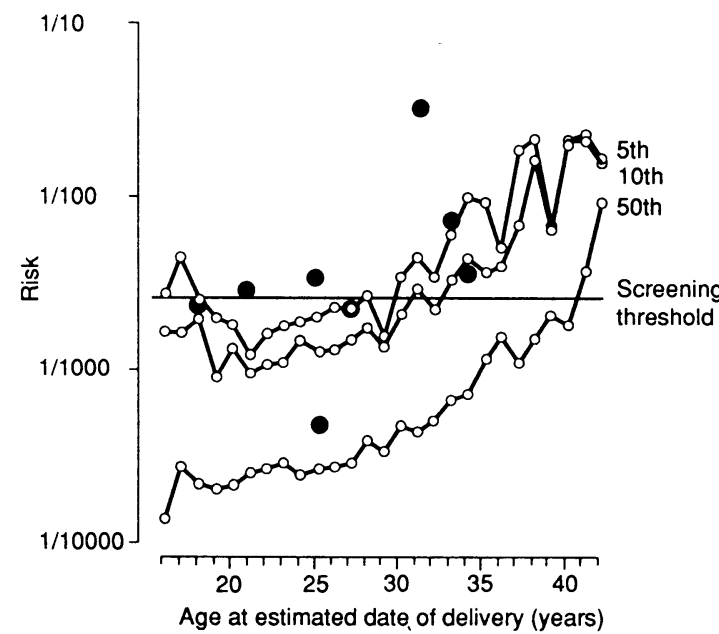

FIG 1-Fifth, 10th, and 50th (median) centiles of risk of Down's syndrome in 3089 pregnancies in women aged 16-42 at the expected date of delivery. Individual risks of women with affected pregnancies are shown by

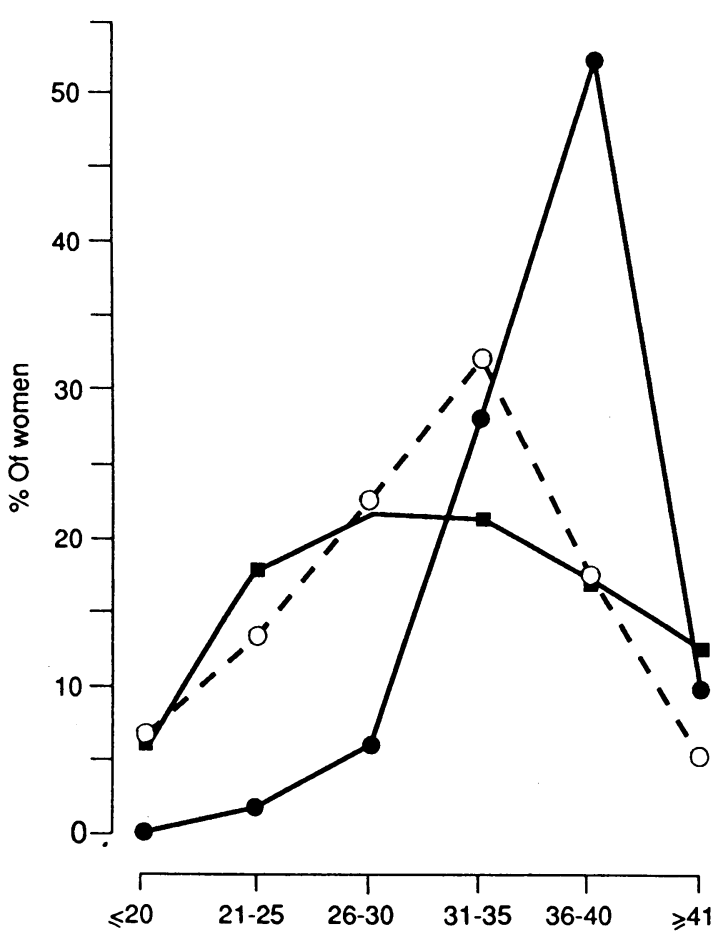

Age at estimated date of delivery (years)

FIG 2-Age distributions of women who had fetal karyotype determination in Tayside, 1988-90 (O) and women who would have been offered screening on basis of individual risk, 1988-90( $(\mathrm{)})$ and expected age distribution of women with affected pregnancies from national $\operatorname{data}(\mathbf{\square})$
Only one of the cases was detected by current screening practices.

Figure 2 shows the age distribution of women in Tayside for whom fetal karyotypes were determined during November 1988 to March 1990 and that of the women who would have been offered karyotype determination on the basis of individual risk analysis. The figure also shows the expected age distribution of women giving birth to babies with Down's syndrome derived from national data. ${ }^{10}$

Three further pregnancies associated with Down's syndrome were recorded in 1990. One in a woman aged 42 was detected by amniocentesis; the two others, in women aged 22 and 27 years were not screened and resulted in live births. When we calculated the individual risk all three pregnancies had risks above the screening threshold of $1: 350$ ( $1: 120$ for 22 year old, $1: 80$ for 27 year old; and 1:90 for 42 year old).

Of the 11 affected pregnancies recorded between November 1988 and December 1990, eight (73\%; 95\% confidence interval $39 \%$ to $94 \%$ ) had risks above the screening threshold. The geometric mean $\alpha$ fetoprotein concentration found in the samples from unaffected pregnancies was 0.99 multiples of the median, and the geometric mean chorionic gonadotrophin concentration in the same samples was 1.05 multiples of the median. In pregnancies associated with Down's syndrome the corresponding figures were 0.68 and 2.08 multiples of the median.

\section{Discussion}

Screening pregnant women for Down's syndrome based on maternal age and genetic history is ineffective in detecting most cases. From 1987 to 1990 Down's syndrome was detected antenatally in seven out of 27 women with affected pregnancies in Tayside.

From November 1988 to March 1990 karyotypes were obtained in $5 \%$ of pregnancies, $62.5 \%$ of which were in women over 35 ; but only $4 \cdot 8 \%$ of births and only one affected pregnancy occurred in this age group. If screening had been done on the basis of individual risks the women with five of the eight affected pregnancies that survived to the second trimester would have been offered screening; karyotypes would have needed to be obtained for $6.3 \%$ of pregnancies. These data accord well with the predictions made by Wald et al. ${ }^{3}$

If data from the three affected pregnancies recorded during April to December 1990 are included screening on the basis of individual risk would have detected eight of $11(73 \%)$ affected pregnancies. A similar degree of success has been reported in retrospective analyses of data from selected specimens from affected and unaffected pregnancies. ${ }^{21-13}$ Macri et al suggested that free $\beta$ chorionic gonadotrophin may be a better marker than the intact molecule, ${ }^{12}$ although their detection rate was identical with ours.

The age distribution of women who would have been offered screening on the basis of individual risk during the study period matched the expected distribution of women having babies with Down's syndrome more closely than did the age distribution of women who had karyotype analysis (fig 2). Screening based on risk analysis would have given a false negative rate of $38 \%$ (27\% with the updated figures for 1990), which compares with a rate of $88 \%$ ( $82 \%$ updated) from screening based on maternal age and genetic history.

Unless resources were increased by $50 \%$ performing amniocentesis and karyotype determination on the basis of calculated risk would be possible only if the automatic right of women aged over 35 to these procedures were removed. ${ }^{2}$ It is therefore important to be able to reassure women over 35 , who are currently almost guaranteed that a fetus with Down's syndrome 
will be detected, that the new test has a similar probability of success. The distribution of medians and individual risk estimates in our study suggests that there is a high probability that women older than 35 with affected pregnancies would be offered screening. This was also found in a recent study by Mancini $e t a l .^{13}$ Furthermore, as the median calculated risk for women over 40 is higher than 1:350 more than half of those women would be offered screening. As only $0.6 \%$ of births are in women over 40 offering amniocentesis and karyotyping to all women in this age group would increase the number of pregnancies requiring screening by only about $0.3 \%$. This would also address the problem that allocating access to karyotype determination on the basis of risk of Down's syndrome alone ignores the possibility of other chromosomal abnormalities in older pregnant women. Other chromosomal disorders are rarer than trisomy 21 and because the risk is less strongly age dependent are less effectively screened by age alone.

The number of younger women requesting amniocentesis because of anxiety might be expected to fall when counselling can include the discussion of a method of individual risk assessment with a high expectation of success in detecting Down's syndrome. This should compensate the increase in screening generated by offering karyotype screening to women aged over 40 without biochemical evidence of their pregnancy being at high risk of Down's syndrome.

This study was supported by Tayside Health Board. We thank Valerie Berthoud, Lyndsey Cuthill, and Shirley Moore for technical help; Sharon Mudie and Lesley Nicol for retrieval of cytogenetic and ultrasonic data; and Margaret Henderson for secretarial help. We also thank the Department of Biochemical Medicine for providing stored serum samples.

1 Cucke HS, Wald NJ, Thompson SG. Esimating a womas risk of having a pregnancy associated with Down's svndrome using her age and maternal serum alpha-fetoprotein level. Brf Obstet (iynaecol 1987;94:387-402.

2 Norgaard-Pedersen B, Larsen SO, Arends J, Svenstrup B, Tabor A. Maternal serum markers in screening for Down syndrome. Clin Genet 1990;37:35-43.

3 Wald NJ, Cuckle HS, Densem JW, Nanchahal K, Canick JA, Haddow JE, et al. Maternal serum screening for Down's syndrome in early pregnancy. BMF 1988;297:883-7.

4 Merkatz IR, Nitowsky HM, Macri JN, Johnson WE. An association between low maternal serum $\alpha$ fetoprotein and fetal chromosome abnormality. $A m \mathcal{J}$ Obstet Gynecol 1984;148:886-94.

5 Bogart MH, Pandian MR, Jones OW. Abnormal maternal serum chorionic gonadotrophin levels in pregnancies with fetal chromosome abnormalities. Diagn 1987;7:623-30.

6 Canick JA, Knight GJ, Palomaki GE, Haddow JE, Cuckle HS, Wald NJ. Low second trimester maternal serum unconjugated oestriol in pregnancy with Down's syndrome. Br f Obstet Gynaecol 1988;95:330-3.

7 Stevenson JD, Chapman RS, Perry B, Logue FC. Evaluation and clinical application of a two site immunoradiometric assay for alpha-1-foetoprotein using readily available reagents. Ann Clin Biochem 1987;24:411-8.

8 Walker EM, Lewis M, Cooper W, Marnie M, Howie PW. Occult biochemical pregnancy: fact or fiction? Br f Obstet Gynaecol 1988;95:659-63.

9 Christie AD. Standards in ultrasound fetal biometry [PhD thesis]. Dundee University of Dundee, 1980.

10 Wald NJ, Cuckle HS. Biochemical detection of neural tube defects and Down's syndrome. In: Turnball AC, Chamberlain G, eds. Obstetrics. Edinburgh: Churchill Livingstone, 1989:269-89.

11 Suchy SF, Yeager MT. Down syndrome screening in women under 35 with maternal serum hCG. Obstet Gynecol 1990;76:20-4.

12 Macri JN, Kasturi RV, Krantz DA, Cook EJ, Moore ND, Young JA, et al. Maternal serum Down syndrome screening: free $\beta$ protein is a more effective marker than human chorionic gonadotropin. Am J Obstet Gynecol 1990;163: 1248-53.

13 Mancini G, Perona M, Dall'Amico D, Bollati C, Albano F, Mazzone R, et al. Screening for fetal Down's syndrome with maternal serum markers - an experience in Italy. Prenat Diagn 1991;11:245-52.

(Accepted 13 fune 1991)
Human Population

Laboratory, California

Public Health Foundation, Berkeley, California 94704-9980, United States

Nancy B Lazarus, MD, research scientist

Richard D Cohen, MA, biostatistician

Diing-Jen Leu, MPH,

research analyst

\section{Human Population}

Laboratory, California

Department of Health

Sciences, Berkeley,

California 94704-9980,

United States

George A Kaplan, PHD,

chief of laboratory

Correspondence and requests for reprints to: Dr Kaplan.

BMF 1991;303:553-6

Change in alcohol consumption and risk of death from all causes and from ischaemic heart disease//

Nancy B Lazarus, George A Kaplan, Richard D/Cohen, Diing-Jen/Leu

\section{Abstract}

Objective-To examine the association between alcohol consumption and mortality from all causes and from ischaemic heart disease with a focus on differentiating between long term abstainers and more recent non-drinkers.

Design-Cohort study of changes in alcohol consumption from 1965 to 1974 and mortality from al causes and ischaemic heart disease during 1974-84.

Setting-Population based study of adult residents of Alameda County, California.

Subjects -2225 women and 1845 men aged 35 and over in 1965.

Main outcome measures-Alcohol consumption in 1964 and 1974 and mortality from all causes and from ischaemic heart disease during 1974-84.

Results - There was a significantly higher risk of death from all causes and from ischaemic heart disease in women who gave up drinking between 1965 and 1974 than in women who continued to drink (relative risk $1 \cdot 72,95 \%$ confidence interval 1.11 to 2.66 , and $2.75,1.44$ to 5.23 , for all causes and ischaemic heart disease respectively). A significant increase in risk was not seen in men who gave up drinking $(1.32,0.87$ to 2.01 , and $0.95,0.41$ to $2 \cdot 20$, respectively). Among men, long term abstainers compared with drinkers were at increased risk of death from all causes and from ischaemic heart disease, though the associations were not significant $(1.40,0.98$ to 2.00 , and $1.40,0.76$ to 2.58 , for all causes and ischaemic heart disease respectively).

Conclusion-Some of the increased risk of death from all causes and from ischaemic heart disease associated with not drinking in women seems to be accounted for by higher risks among those who gave up drinking. Men who are long term abstainers may also be at an increased risk of death. The heterogeneity of the non-drinking group should be considered when comparisons are made with drinkers.

\section{Introduction}

Several studies have described a J-shaped association between intake of alcohol and risk of cardiovascular disease, with the lowest risk among light drinkers and higher risks among non-drinkers and heavy drinkers. ${ }^{1-8}$ The observation that non-drinkers have a higher incidence of cardiovascular disease than light drinkers has led to light drinking being characterised as "protective." This characterisation is not without biological plausibility given the observations of increased concentrations of high density lipoprotein cholesterol with increased alcohol consumption. ${ }^{34-11}$ Also, Meade et al have suggested that alcohol's antithrombotic effects may be responsible for this protective effect. ${ }^{12}$

There is, however, another possible explanation for the effect. Most analyses treat non-drinkers as a homogeneous group, but it is likely that non-drinkers constitute an extremely heterogeneous group consisting of life long abstainers and also former drinkers, who may have a higher risk of death. ${ }^{13.14 a}$ If these subgroups differ with respect to risk of cardiovascular 